\title{
Risk perception and behaviour change after personal vaccination for COVID-19 in the USA
}

\author{
Jayson S. Jia ${ }^{1 *}$, Yun Yuan ${ }^{2 *}$, Jianmin Jia ${ }^{3,4}$, Nicholas A. Christakis ${ }^{5}$ \\ ${ }^{1}$ Faculty of Business and Economics, The University of Hong Kong, Hong Kong SAR, \\ China. \\ ${ }^{2}$ School of Economics and Management, Tsinghua University, Beijing, China. \\ ${ }^{3}$ Shenzhen Finance Institute, School of Management and Economics, The Chinese University \\ of Hong Kong, Shenzhen, China. \\ ${ }^{4}$ Shenzhen Institute of Artificial Intelligence and Robotics for Society, Shenzhen, China. \\ ${ }^{5}$ Yale Institute for Network Science, Yale University, New Haven, CT, USA. \\ *J.S.J. and Y.Y. contributed equally to this paper \\ †Corresponding authors: jjia@hku.hk (J.S.J.), jmjia@cuhk.edu.cn (J.J.), \\ nicholas.christakis@yale.edu (N.A.C.)
}

Keywords: COVID-19 vaccine, behavioural health, risk perception and decision making, personal protective behaviours, socioeconomic recovery

\begin{abstract}
Although vaccines are crucial for giving pandemic-stricken societies the confidence to return to socioeconomic normalcy, vaccination may also induce laxity in personal protective behaviours (e.g., handwashing, facemask use, and social distancing). We use the quasiexperimental context of the COVID-19 vaccine rollout across the United States to quantify the impact of different stages of personal vaccination on people's risk perceptions, daily activities, and risk mitigation behaviours, which we measure in a three-wave national panel study $\left(\mathrm{N}_{\text {wave-1 }}=7,358, \mathrm{~N}_{\text {wave-2 }}=3,000, \mathrm{~N}_{\text {wave-3 }}=2,334\right)$ from March to June, 2021, and validate using state-level vaccination, infection, and human mobility data. Socioeconomic behaviour recovered and risk-mitigation declined as vaccination progressed with heterogeneous effects depending on demographics and infection history; risk perception declined and socializing rebounded after only partial vaccination and communal activities recovered after full vaccination. We further use a utility theory framework to explain the risk-wellbeing trade-offs underlying different behavioural changes.
\end{abstract}


Mass vaccination programs are a turning point in the COVID-19 pandemic when societies shift from pandemic containment towards socioeconomic recovery ${ }^{1}$. Although the vaccination gives people the confidence to lead normal lives, the protection they afford may lead to unintended consequences for human behaviour. In particular, by lowering the perceptions of COVID-19 risk, vaccines may reduce people's protective vigilance, which may increase epidemiological danger in the face of new variants of COVID-19.

Previous studies have shown that by lowering perceived risk, risk-mitigation measures can also facilitate compensatory risky behaviour, which can offset some of their benefits. For example, introducing improvements in automobile safety may give rise to riskier driving; medications providing some protection against HIV can lead to more risky sexual behaviour; taking cholesterol medication can provide false assurance and lead to increased caloric and fat intake over time ${ }^{2-7}$. Whether analogous behaviours arise for COVID19 vaccines is an open question. Although people are generally conservative with respect to risky behaviors ${ }^{8-10}$, other factors, such as adaptation to risk, normalization of COVID-19, and pandemic fatigue may promote risk compensation behaviours after vaccination ${ }^{11-12}$. Indeed, for many people who want a COVID-19 vaccine, the desire to "return to normal" is paramount ${ }^{13-14}$, which may lead to less vigilant adherence to non-pharmaceutical interventions (NPIs). Such behavioural trends are especially risky in the face of highly contagious strains of COVID-19 such as delta and omicron, which are difficult to contain using vaccination alone ${ }^{15-17}$.

We collected national panel data to investigate the effect of vaccination on behaviour and risk perceptions across time as COVID-19 vaccines became available in the USA. We measured people's risk perception, daily activities (e.g., socializing, commerce), and risk protection measures (e.g., handwashing, mask wearing, and social distancing). Vaccine coverage was $14.9 \%$ at the start of the first-wave survey, and $49.6 \%$ at the end of the thirdwave survey. We later validate the survey results against location-specific CDC vaccination statistics and Google and Facebook human mobility measures.

Although it is obvious that vaccines provide significant protection against COVID-19 infection, how much they lower subjective risk perception, and how this translates into behavioural change as people progress through the different stages of personal vaccination is not clear. We explore several issues. First, we investigate the degree to which different socioeconomic behaviours recover at different stages of vaccination. Second, we examine if and how much protective behaviours recommended by the CDC at the time concurrently decline. Third, we measure the impact of vaccination on perceived risk, which could explain 
both forms of behavioural change, and explore demographic heterogeneities. In addition, our analysis empirically separates the impact of getting vaccinated, from the impact of herd protection (i.e., percentage of local population vaccinated) or environmental risks (local prevalence of COVID-19 cases) on perceived risk. Unlike many other risks, epidemiological risk is socially determined, which adds a social and mobility layer of risk ${ }^{18-21}$. In the case of vaccination, a person's assessment of risk may thus relate to the rate of vaccination among others around them. Fourth, we validate our survey results using Google and Facebook mobility data from the corresponding dates and locations.

Finally, to understand the possible motivations behind post-vaccination behavioural changes, we model the subjective risk-value trade-offs for each individual-level behavior ${ }^{22-24}$. Recent research also shows that perceived COVID-19 risk closely predicts compliance with public health guidelines and that people's preferences for locations are based on a trade-off between transmission risk and social benefits ${ }^{25}$. Since vaccines do not completely eliminate COVID-19 risk, vaccinated individuals must still make subjective trade-offs between the risks and benefits for different behaviours. Normal socioeconomic behaviours increase wellbeing but may also be risky; protective behaviours decrease risk but come at some cost to wellbeing. By estimating the trade-offs that people make between wellbeing and risk for each activity, we can better understand why people engage in epidemiologically suboptimal behaviours, and predict which behaviours are easier (or harder) to promote (or discourage). Such insights may facilitate more effective risk communications that balances behavioural normalization and risk mitigation to achieve an optimal "target level of risk" in human behaviour $^{3-4,9}$.

\section{Data}

The US national vaccination campaign provides a "quasi-experiment" where respondents progress through different stages of vaccination or remain unvaccinated (which serves as a reference state in our main analyses). We conducted a three-wave panel survey in the United States between March 11 and June 20, 2021, which yielded a valid sample of 2,902 after two waves and 2,334 after three waves (see Methods for details).

Quasi-experimental analysis is widely used in social science to make limited causal inferences where random assignment is impossible or unethical ${ }^{26}$. We use Difference-inDifference (DiD) models, which exploit the within-subjects change in respondents' vaccination status across time, to estimate the impact of vaccination on behaviour, risk perception, and wellbeing. In contrast to a panel regression model, a DiD model considers 
change in behaviour against a reference group (e.g., individuals who are unvaccinated during the event time), which mitigates the effects of trend changes and extraneous factors, and also differences out time-invariant unobserved individual characteristics such as vaccine hesitancy. We first use a simple DiD model for ease of interpretability. We then use a flexible event-study DiD framework that considers variation in vaccination event times (i.e., staggered rollout) to model heterogeneous treatment effects.

Besides recording respondents' vaccination status (number of shots, vaccine type), we also recorded their zip codes (validated against IP-geolocation), which allow us to control for state-level vaccination rate and COVID-19 case count. This statistically separates the impact of individual-level changes in vaccination status from perceived herd immunity benefits as more people are vaccinated in the region.

We measured psychological wellbeing ("happiness"), risk perception (overall threat, probability of infection, consequence of COVID-19 infection), engagement in preventative behaviours (such as facemask use, staying at home, social distancing, washing hands, etc.), and daily activities (such as dining indoors, social gatherings, meeting strangers, visiting crowded places, taking rideshare or public transport, traveling etc.) in the preceding four weeks, and expected relative frequency of the same behaviours in the next 4 weeks, as dependent variables (see Table S1 for survey questions). We also include demographics (age, gender, ethnicity, income, education, political leaning, location, etc.) as covariates.

We tested the external validity of the survey data against CDC vaccination data and behavioural digital trace data ${ }^{27}$, including Google and Facebook mobility indices (Fig. 1a-f). First, the number of vaccinated survey respondents is highly correlated with the vaccinated population at the state-level across time (Pearson's $r=0.945, p<0.001$ across three waves of surveys). Second, self-reported recent daily activities are significantly correlated with Google mobility index measures of change in different location-category visits (versus pre-pandemic baseline; Pearson's $r=0.520, p<0.001$ across all states; $r=0.721, p<0.001$ for the top 16 states in our sample, which covers $70 \%$ of the total sample size). Our survey stay-at-home measure is also significantly correlated with Google's residential mobility index (measuring a residential change in duration), $r=0.621, p<0.001$ across all states; $r=0.802, p<0.001$ for the top 16 states). Finally, we repeated the analyses using Facebook mobility indices "Change in Movement" and "Stay Put", which are significantly correlated with self-reported recent daily activity levels in our survey (For Change in Movement, Pearson's $r=0.643, p<0.001$ across all states; $r=0.817, p<0.001$ for top 16 states in our survey samples; for Stay Put, $r=$ $-0.561, p<0.001$ across all states; $r=-0.674, p<0.001$ for the top 16 states). 
We conducted three sets of analyses. Firstly, we use a simple DiD model (1) to test the parallel trend assumption and estimate the short-term impact of vaccination status on behavioural changes. Secondly, we use an event-study model (2) to examine how different stages of vaccination affect behaviour and risk perception. We further extend the analysis by considering infection history. Finally, we use a utility-theory framework to test if differences in risk-wellbeing trade-offs across behaviours can explain heterogeneities in post-vaccine behavioural change.

For our analyses, we classify survey respondents into 7 groups based on their vaccination status over the three waves of surveys (see Table 1 for classification; Table S2 for descriptive statistics; Table S3 for demographics by group). Overall, behavioural and perceptual measures for all 7 groups have similar relative values and patterns (Fig. S1).

\section{Difference-in-difference Analysis and Parallel Trend Test}

We first use a simple DiD model (1) to estimate the short-term impact of vaccination on behavioural and perceptual changes, and simultaneously test the parallel trends assumption. Here, we limited the sample to groups 1-3, who were all unvaccinated during waves 1 and 2. We modelled the treatment effect of full or partial vaccination by using group 3 or 2 as the treatment group, respectively (by the third wave, group 3 received two doses of a two-shot vaccine or the one-shot Johnson \& Johnson vaccine; group 2 received one dose of a two-shot vaccine). For both analyses, group 1 (who were unvaccinated over all three waves of surveys) is the reference group; this yields conservative results since group 1 had a higher baseline level of activity, lower perceived risk of COVID-19, and lower propensity to engage in preventative behaviours (Tables S4 and S5). By comparing the average change in the outcome variable over three time periods (waves), DiD model (1) uses waves 1 and 2 to test the parallel trend assumption, and uses wave 3 to identify the treatment effects.

We also use the coarsened exact matching (CEM) approach to improve identification by reducing sample imbalance in covariates between the treatment (i.e., vaccinated) and reference (i.e., unvaccinated) groups. We used age, gender, income, and political partisanship as matching variables, since they may be major factors behind vaccination decisions (and group membership).

Parallel trend test. The parallel trend assumption is the most critical assumption for DiD analysis, and requires that in the absence of treatment, the difference between the treatment and reference group is constant over time. In model (1), we use waves 1 and 2 as a parallel trend test for treatment groups 2 and 3 against reference group 1 (since all three 
groups are unvaccinated during waves 1 and 2); if the parallel trend assumption is satisfied, the estimated parameter $\beta_{1}$ of the interaction term Treat $_{i} \times W_{\text {ave }} 2_{t}$ should not be statistically significant during this pre-treatment period.

We find that this is the case for both treatment groups. When group 3 is the treatment group, the estimated parameter of $\beta_{1}$ is small and not statistically significantly different from zero for every measure except for handwashing (see Fig. 2a). Similarly, when group 2 is the treatment group, the estimated parameter $\beta_{1}$ is not significant for any measures (see Fig. $\mathrm{S} 3 \mathrm{a})$. Overall, the trends of both treatment groups are not significantly different from the reference group in the pre-treatment period for all measures except for handwashing (for group 3).

Vaccination treatment effects. $\beta_{2}$, the parameter of the interaction term Treat $_{i} \times W a v e 3_{t}$ quantifies the effect of vaccination status on behaviour or perception in the third wave (relative to unvaccinated group 1). The interaction carries different meaning depending on treatment group; Group $3 *$ Wave 3 tests the impact of full vaccination (see Tables S4a-c); Group 2*Wave 3 tests the impact of partial vaccination (see Tables S5a-c). To present results more intuitively, we transformed estimated coefficients into percentage changes (e.g., $17.06 \%=e^{0.1871}-1$ for risk perception of infection), in Figs. 2 and S3 (right panels).

After full vaccination (Fig. 2), respondents' risk-probability perception declined $17.1 \%(p=0.006)$ and socioeconomic activities increased by $11.5 \%$ overall $(p=0.006)$; for example, visiting young social ties increased $21.7 \%(p=0.017)$ and shopping increased $19.8 \%$ ( $p=0.001)$. Communal activities did not immediately recover, although respondents planned to engage in almost every socioeconomic activity in the next 4 weeks (overall increase of $21 \%, p<0.001)$ except for visiting crowded places and going to the workplace. This trend of socioeconomic behavioural recovery was accompanied by an immediate decline in risk-vigilance; propensity for risk mitigation behaviours decreased by $-8.68 \%$ overall ( $p=$ $0.033)$; handwashing and social distancing declined by $-12.4 \%(p=0.005)$ and $-15.5 \%(p=$ 0.027), respectively (we urge caution in interpreting the decline in handwashing, since parallel trends assumption was not satisfied for this case).

An analogous analysis with group 2 as the treatment group) suggests that partial vaccinations was enough to generate limited behavioural recovery (Fig. S3); socioeconomic behaviours increased by $13.5 \%$ overall $(p=0.012)$, although this was largely driven by statistically significant increases in consumption-related activities and social interactions. 
Besides for increased intentions to visit elderly social ties, future planned behaviours did not significantly increase after partial vaccination. Protective behaviours also did not change significantly after partial vaccination. However, we urge caution in interpreting these results, since group 2 had a small sample size; furthermore, the treatment effects of group 2 and 3 may not be comparable since they were modelled separately.

Next, we address these limitations with an event study model that exploits the full sample, and also models the temporal effects of vaccination, which tests whether the treatment effect of vaccination status grows stronger at more advanced stages of vaccination.

\section{The Temporal Effects of Vaccination over Different Stages}

We use an event study model to quantify the dynamic treatment effects of different vaccination statuses using data from groups 1-6. The vaccine event study DiD model (2) allows us to aggregate data from different groups according to their vaccination status (resulting in more samples for each status) to simultaneously estimate the average treatment effects for three different stages of vaccination: 1) "just vaccinated" (with one or two doses), 2) "fully vaccinated" (two weeks passed since full vaccination course, as defined by the $\mathrm{CDC}$ ), and 3) "post-vaccinated" (which we define as someone who has been vaccinated for two waves of surveys); $\beta_{k}(k=2,3,4)$ are estimated treatment effects for the three stages of vaccination. In this analysis, the reference group are the all unvaccinated individuals at the corresponding wave of survey. We also include a pre-treatment dummy, $\beta_{1}$, for the wave prior to vaccination; estimating this parameter directly tests the parallel trends assumption.

We estimated model (2) for each of the 39 behavioural or perceptual measures (see Fig. 3 and Tables S7a-c for results). The estimated parameters of the pre-treatment dummy $\beta_{1}$ are very small in magnitude and statistically indistinguishable from zero for all behaviours and perceptions (average $p$-value $=0.554$; see Fig. 3A). Overall, the results provide no evidence of differential behavioural trends between vaccinated and unvaccinated individuals before vaccination.

We investigated the dynamic effects of vaccination on recipients' behaviours and perceptions over the three stages of vaccination (Fig. 3). Overall, the magnitude of behavioural recovery increased (with an especially strong rebound for social activities and shopping), and the propensity for protective behaviours declined (with a dramatic reduction in staying at home) at more advanced stages of vaccination. Relative to the unvaccinated baseline, vaccinated people's risk-probability perception declined by $-10.83 \%(p=0.022)$, - 
$22.71 \%(p<0.001)$, and $-23.94 \%(p<0.001)$ at the just vaccinated, fully vaccinated, and post vaccinated stages, respectively. Analogously, overall protective behaviours declined by $5.07 \%(p=0.045),-11.55 \%(p<0.001)$, and $-17.68 \%(p<0.001)$; overall recent activities increased $10.08 \%(p=0.002), 21.99 \%(p<0.001)$, and $34.78 \%(p<0.001)$; and overall future planned activities increased by $14.57 \%(p<0.001), 32.86 \%(p<0.001)$, and $50.98 \%$ $(p<0.001)$, respectively, at the three stages.

In addition, we observe time-specific effects; individuals' overall propensity to engage in protective behaviours declined in wave 3 . Since we control for vaccination status, epidemiological context, and demographic characteristics, changes in the coefficients for time (i.e., wave-2 and wave-3 dummies) may reflect temporal trends such as adaptation to risk and pandemic fatigue ${ }^{12}$. We observe a decline in facemask use during wave-2 $(-5.27 \%, p=0.042)$ and wave-3 $(-22.03 \% p<0.001)$, and a decline in social distancing $(-10.05 \% p=0.019)$ during wave-3. However, handwashing increased significantly during waves 2 and 3. Overall, these effects had a smaller parameter value and effect than vaccination for most protective behaviours.

Other heterogeneities. Female, lower income, and Democrat-leaning respondents perceived more risk and engaged in more protective behaviours and fewer daily activities relative to male, higher income, and Republican-leaning respondents, respectively. Older people perceived the risk-consequence of infection as higher, and the risk-probability of infection as lower, and engaged in more protective behaviours and fewer daily activities relative to younger people.

Community effects. We also included state-level vaccination rate and COVID-19 infection cases for the preceding two weeks as control variables. Respondents engaged in significantly more daily activities $(p<0.001)$ if their state's vaccination rate increased. Specifically, state vaccination had a significant positive effect on going to crowded places ( $p$ $=0.001)$, visiting older and younger social ties ( $p=0.006$ and $p=0.04$, respectively), going to restaurants $(p=0.008)$, inviting people home $(p=0.004)$, and traveling $(p=0.035)$. Statelevel vaccination rate had no significant overall effect on individual protective behaviours. Recent infection cases (preceding two weeks) had a significant negative effect on shopping ( $p$ $=0.014)$; however, infection rate did not have a significant overall effect on daily activities. Overall, state-level vaccination and infection did not materially change our other results. Although we urge caution in interpreting this null effect (particularly since state vaccination rate may be confounded with other factors), it is nonetheless noteworthy that community effects were small compared to the very significant personal vaccination effects. 
An integrated analysis including infection history. Since COVID-19 infection history may correspond to risk attitudes and certain behavioural propensities, we excluded selfreported infected respondents and only included uninfected respondents in our primary analysis. As a robustness check, we tested an integrated model (6) (See SI, part 5), which includes interaction effects between infection and vaccination state; both uninfected and infected respondents were included in this model. The infection rate among survey respondents is $7.16 \%$; the official US infection rate at the corresponding time was $9.7 \%$.

Model (6), and the inclusion of infected respondents, replicated the results of model (2) (see Figs. S9a-b); treatment effects for just vaccinated, fully vaccinated, and postvaccinated were similar; we also observed the same temporal pattern for stage of vaccination, whereby socioeconomic behavioural recovery increased more and protective behaviours declined more at more advanced stages of vaccination. In addition, the infection history and treatment interaction terms had no significant effects (Fig. S9b), which shows that vaccine state treatment effects were similar for both infected and uninfected respondents. The only exception was perceived threat; infected respondents perceived significant less threat than uninfected respondents before and after vaccination.

\section{Risk-Wellbeing Trade-offs}

We used a utility theory framework to investigate why some behaviours decline while others increased after vaccination (see Methods models (3)-(5)). In decision-making under risk, there is a fundamental trade-off between risk and reward-value ${ }^{22}$. The COVID-19 pandemic introduced significant risks related to behaviours that were once considered riskless, and increased the utility of risk-prevention behaviours that were once seen as unnecessary. Vaccination likely engenders the opposite effect. Since we measure both utility (with our risk and happiness assessments) and behaviour, which are the left- and right- handside variables of a utility model, respectively, we can statistically estimate how much engaging in different behaviours affects people's perceived risk and wellbeing. We also model the trade-off between risk and wellbeing for individual behaviours.

Utility weighting of behaviours after vaccination. We first use a DiD model to test how much daily activities and preventative behaviours contribute to utility (well-being or perceived risk) after vaccination. Here, we use a two-wave panel of uninfected respondents for easier interpretability (see Table S10 for group composition), and compare fully vaccinated to unvaccinated respondents(Table S11). Happiness is positively associated with daily activities $\left(a_{1}=0.055, p=0.004\right)$ but not significantly associated with preventative 
behaviours. Perceived threat of COVID-19 is negatively associated with daily activities $\left(a_{1}=\right.$ $-0.159, p<0.001)$ and positively associated with preventative behaviours $\left(b_{1}=0.576, p<\right.$ 0.001 . However, the interaction between preventative behaviours and vaccination is a significant positive predictor of happiness $\left(a_{3}=0.021, p=0.019\right)$, and a negative predictor of perceived risk $\left(b_{3}=-0.024, p=0.073\right)$. Overall, vaccination can increase the utility of preventative behaviours both by increasing wellbeing and by reducing perceived risk. We find no analogous effect for daily activities, suggesting that vaccination does not affect the utility of daily activities.

Risk-Wellbeing trade-offs. We next estimate the trade-off between risk and wellbeing for each individual behaviour. We extract the trade-offs by testing whether different behaviours mediate the inherent negative relationship between risk and wellbeing (Table S12). Fig. 4 plots the indirect effect of perceived threat on each behaviour on the $\mathrm{x}$-axis and the indirect effect of behaviour on happiness on the y-axis. We find that while preventative behaviours and daily activities generally have positive and negative relationships, respectively, with perceived risk, they have heterogenous relationships with happiness. Indeed, some behaviours have a less positive effect on happiness without the benefit of having a less negative effect on risk; in other words, they offer worse risk-value trade-offs. For example, all preventative behaviours have a similar relationship with risk, but whereas handwashing, ordering online, and facemask have no significant relationship with happiness, we observe that staying-at-home negatively affects happiness significantly. Similarly, many daily activities do not have a significant (positive) indirect effect on happiness; these include going to crowded places, workplaces, and movie theatres, which may be considered unnecessary (or substitutable) during a pandemic. This is consistent with our earlier analysis showing that the same behaviours recovered less after vaccination (Fig. 3). We replicated these findings using an alternative analysis that adds happiness and threat to model (2) (Table S8a-c, Fig. S10).

Probability-consequence trade-offs. Finally, we adapt Model (5) to explore whether vaccination affects how people construct their perceptions of COVID-19 risk. In particular, risk can be decomposed into two major components: consequence (i.e., the outcome if a risk materializes), and probability (i.e., susceptibility) that may be differentially weighted ${ }^{28}$. We test whether vaccination changes how the consequence versus probability of COVID-19 infection informs overall risk (see Methods; results in Table S13). We compare fully vaccinated groups A3 and A4 with the unvaccinated group A1 (using two waves of survey 
data).

We find that the perceived threat of COVID-19 is determined primarily by the consequence of getting COVID-19, which is weighted 3.4 times more than probability of infection (i.e., susceptibility). The vaccinated groups have a higher baseline risk perception of COVID-19 compared to the unvaccinated group, $c_{1}=0.162(p<0.001)$. After vaccination, the vaccinated group weights consequence less $\left(b_{3}=-0.043, p=0.001\right)$, but there is no significant interaction for probability. This shows that perceived threat declined because people mainly believe that vaccines reduce the consequences of COVID-19 infection. The same analysis for partially vaccinated people (group A2 versus group A1) yielded no significant main or interaction effects, which shows that partial vaccination did not significantly change risk perception (despite objectively providing some protection).

\section{Discussion}

When implementing mass vaccination campaigns, it is essential to understand not just their clinical, but also their behavioural impact. In the case of COVID-19 vaccinations in the United States, full vaccination generates several behavioural spillover effects. We find that although vaccines have the intended effect of encouraging normalized socioeconomic behaviour (some more than others), they also reduced the propensity to engage in protective behaviours over time. The decline in preventative behaviours is reminiscent of the "Peltzman Effect", where people's behaviours become relatively riskier after receiving increased protection ${ }^{2}$. Indeed, we find that social activity increased even after partial vaccination. Our statistical approach leveraged the longitudinal, quasi-experimental context of the vaccine rollout; we used three waves of panel data (which we cross-validated against CDC vaccination and Google and Facebook mobility data) to quantify the impact of the US vaccination campaign on residents' risk perceptions, happiness, and behaviours. This withinsubjects approach also differences out individual-level heterogeneities and trend effects, and removes many biases inherent in other approaches to this topic, all of which is important for informing policy decisions.

Respondents' risk perception declined and daily activities increased in a graded pattern as they transitioned from an unvaccinated to partially-, fully-, and post-vaccinated state. The most significant behavioural changes occurred immediately after full vaccination, and gradually increased thereafter. And not all behaviours recovered to the same degree: social needs such as meeting friends or family and offline commerce activities such as shopping and going to a restaurant increased the most. Indeed, individuals significantly 
increased social activities even after partial vaccination. Communal behaviours, such as taking an airplane, taking rideshare or public transport, and going to the movies or workplace were prioritized less, and taking rideshare or public transport only increased significantly during the post-vaccinated stage. Overall, the vaccine-abetted behavioural recovery was uneven, particularly for communal activities that have home-based substitutes (e.g., teleworking or movie streaming, which have become normalized).

We also tested for community effects, i.e., percentage of local population vaccinated, which had a positive significant effect on daily activities but had no significant effect on individual protective behaviours based on our models. Although it is possible that our sample did not pick up community effects (particularly since neighbourhood-level vaccination is difficult to measure reliably), it is notable that its effect is dwarfed by the highly significant impact of personal vaccination. We may thus conclude that the strongest behavioural effects came from (perceived) personal protection; this is also rational in a social environment where vaccination rates were less than 50\% (which was the case for most of our respondents, over time).

However, lower perceived risk after vaccination also led to a reduction in preventative behaviours (NPIs). Staying at home, handwashing, and stockpiling declined significantly but gradually across the different stages of vaccination. Although the overall propensity for preventative behaviours decreased significantly after vaccination, people maintained NPIs such as facemask use and social distancing.

The propensities to engage in protective behaviours and daily activities have a negative relationship $(r=-.458, p<.001$, in wave-1); protective behaviours tend to decline as daily activities recover, particularly in the context of "pandemic fatigue"12-13. The negative relationship between behavioural normalcy and risk-mitigation makes risk communications and management challenging during vaccine rollouts. For example, in July 2021, the US CDC primarily communicated that "people who have been fully vaccinated can start to do some things that they had stopped doing because of the pandemic." However, a month later, during the upsurge in Delta variant transmissions in August 2021, the CDC urged even fully vaccinated people to continue practicing everyday preventive behaviours in order to maximize protection for themselves and others.

Only $25 \%$ of respondents maintained risk-mitigation measures while normalizing daily activities, which shows the inherent difficulty of balancing risk-mitigation with return to normalcy. Psychology research suggests that normal and preventative behaviours may fall under two distinct 'behavioural modes ${ }^{29}$, and people's evaluations of fear and positive 
reward may even have distinct neurological underpinnings ${ }^{30}$. Worrisomely, this behavioural pattern may contribute to increased COVID-19 transmission even after vaccination. While it seems unlikely that those behaviours can completely undo the impact of the vaccine, future work can quantify the impact of such an upsurge in risky behaviours.

As an exploratory study, we combined vaccination, COVID-19 case count, and Facebook human mobility data ${ }^{27}$, which we use as a county-level measure of (normalized) daily activities, from March 27 to October 31 (Fig. S7, Table S14-15). A naïve analysis shows that locales with more vaccinations actually had higher case count; however, this relationship is mediated and suppressed by mobility. Once mobility is included in the mediation analysis, vaccination has a negative relationship with case-count. These relationships highlight the significant interplay between vaccination, behaviour, and infection case-count, which are consistent with the "Peltzman Effect" documented in our survey results.

Our risk-value trade-off model helps identify which daily activities and NPIs policymakers may restrict or mandate at lower political and social costs (i.e., which behaviours have a better risk-value trade-off), particularly in the event of an uptick in infections. The optimal policy outcome is to encourage high-utility, lower-risk daily activities while maintaining low-utility-cost preventative behaviours. To this end, our risk model quantifies how people trade-off risk and wellbeing for different behaviours. For example, we find that although most daily activities increase both happiness and risk, many communal behaviours increased risk without significantly increasing happiness (which explains their lower rates of recovery). Furthermore, we show that only staying at home reduced happiness, while facemask wearing, hand washing, and social distancing did not have a significant negative effect on happiness. Indeed, handwashing is a relatively easy behaviour to promote but declined the most after vaccination, while social activities with friends and family increased even after partial vaccination despite being a major source of transmission risk ${ }^{17}$.

Overall, the priority of behavioural recovery is determined by people's risk and wellbeing trade-offs. Vaccination encourages both behavioural recovery and decreased vigilance, which highlights the need to for behavioural risk management even after crossing the threshold of getting a vaccine.

\section{Methods}

Dataset description. We used Qualtrics, a major online survey platform, to recruit a threeperiod survey panel. Membership of Qualtrics's online participant pool is voluntary and 
respondents are incentivized with monetary payment. We conducted the three survey waves on a rolling basis; each survey was approximately 8-10 minutes long.

The first wave was conducted between March 11 to April 12, when 14.9\% and 29.6\% of the US population, respectively, were covered (doses administered for each vaccine type divided by the number of doses required for full vaccination) by the vaccination campaign; the second wave was conducted between April 16 and May 5, when 31.6\% and 38.8\% were covered, respectively. The third wave was conducted between May 27 to June 20, when $45.3 \%$ and $49.6 \%$ of the US population were covered, respectively.

The 3,091 respondents in the second wave were recruited from people who completed the first wave survey $(\mathrm{N}=7,358)$. We removed 91 invalid respondents from the sample $(54$ for inconsistent vaccination status reporting; 36 who reported being on one dose during both waves 1 and 2; 1 outlier who reported being 100 years old) and 98 respondents with invalid zip codes (which cannot be merged with state-level case and vaccination data), which yielded a final $\mathrm{N}=2,902$ for the second wave. 204 respondents reported having previously been infected with COVID-19; the remaining 2,698 respondents reported no infection history or were not sure. We focus on uninfected respondents in our main analysis, but conduct secondary analysis including both uninfected and infected respondents to test for possible heterogenous treatment effects (Figs. S9a-b).

Respondents in the third wave were recruited from the respondents who completed the first and second wave surveys. After removing 26 respondents for inconsistent reporting of vaccination status and 11 respondents with invalid zip codes, the three-period panel contained 2,334 valid respondents, among whom 2,167 reported no history of COVID-19 infection.

The panel consisted of adult residents of the United States and was demographically representative in terms of gender, age group, and geographical region. In the survey sample, White (83.9\%) respondents were relatively overrepresented, as were older individuals. However, our DiD analyses, which differences out individual-level heterogeneities, mitigates the influence of such biases.

In our main analysis, we classify respondents into 7 groups based on vaccination status across the three waves of surveys. Table 1 provides a summary of those groups with the total numbers of uninfected respondents across the three waves, and their demographic features are summarized in Table S2. The three numbers in the bracket in the second column represent different vaccination states in the three waves. For instance, for Group 4, the vaccine state $(0,1,2)$ means no vaccination in wave 1 , but one dose received in wave 2 and 
second dose received in wave 3 . We also have an alternative grouping to classify our data into 4 groups A1-A4 for a two-wave panel data analysis of risk-wellbeing trade-offs.

Survey questions. For ease of reporting, our analyses in the main text combines these measures into indices of overall protective behaviour (4 items), recent daily activities (13 items), and future activities (13 items) which are supported by a factor analysis and have reliability values of (Cronbach's $\alpha$ in wave-1) $0.83,0.93$, and 0.93 , respectively (See SI for details).

Difference-in-difference models. By comparing the average change in the outcome variable over time periods (waves) for the treatment (i.e., vaccinated) groups against the reference (i.e., unvaccinated) group, a DiD model mitigates the effects of trend changes and extraneous factors, and also differences out time-invariant individual characteristics ${ }^{26}$.

Model 1. For ease of interpretation, we first use a simple DiD model to estimate the effects of vaccination, time (wave), and their interaction on a series of dependent variables, including psychological wellbeing, protective behaviours, recent activities, and planned (future) activities. The model also tests whether the parallel trend assumption is satisfied.

$\log \left(y_{i, t}\right)=\alpha_{1}$ Treat $_{i}+\alpha_{2}$ Wave $_{t}+\alpha_{3}$ Wave $_{t}+\beta_{1}$ Treat $_{i} \times$ Wave $_{t}+$ $\beta_{2}$ Treat $_{i} \times$ Wave $_{t}+\gamma$ Controls $_{i}+\mu_{i}+\varepsilon_{i, t}$

where $y_{i t}$ represents the value of each survey item for respondent $i$ at time $t$. Treat $_{i}$ is a dummy variable; if a respondent belongs to treatment Group $i$ ( $i=2$ or 3 ), the value is 1 , otherwise it is $0(i=1)$. Wave $2_{t}$ and $W a v e 3_{t}$ are also dummy variables; the value is 1 if the data is from the second wave or third wave, respectively, otherwise it is 0 . The interaction between treatment and wave carries different meaning depending on time period; Treat $_{i} \times W$ ave $2_{t}$ allows us to examine whether the parallel trend assumption for DID is satisfied in the pre-treatment time period before vaccination ${ }^{31}$ Treat $_{i} \times W a v e 3_{t}$ tests the treatment effect (i.e., full or partial vaccination) on behavioural and perceptual measures in the third wave. Controls $i$ are a set of control variables, including local COVID-19 rate (cumulative case count in a 14-day window divided by State population) and local vaccination rate (percentage people having gotten one or more shots in each State). $\alpha_{j}, \beta_{k}$ and $\gamma$ are parameters to be estimated $(j=1,2,3 ; k=1,2)$. We also control for the entityfixed effect $\mu_{i}$ to mitigate the impact of omitted time-invariant variables such as individual- 
specific characteristics.

We use the coarsened exact matching (CEM) approach to reduce selection biases arising from pre-treatment differences between the treatment (vaccination) and reference (no vaccination) groups. CEM coarsens each covariate into meaningful bins, matches observations based on these bins, and then retains the covariates' original values for analysis $^{32}$. Compared with other matching methods such as propensity score matching, CEM can generate matched data sets with lower imbalance ${ }^{322}$. The demographic variables used for matching include age, gender, income level, and political partisanship since those factors may influence whether respondents are treated or untreated (i.e., vaccinated or unvaccinated). For instance, people aged 65 and older had priority during the US vaccine rollout and Democrats were more willing to be vaccinated than Republicans ${ }^{33}$.

Model 2. To estimate the treatment effects (of change in vaccination status) for all groups across the 3 survey waves and account for staggered treatment adoption, we use a flexible event-study DiD framework ${ }^{34-36}$, which translates the average treatment effects of group and time from calendar time (survey waves) into event time (vaccination phase). We use the following model to estimate the average treatment effects (i.e., impact of vaccination status) aggregated across different groups based on their stage of vaccination:

$\log \left(y_{i, t}\right)=\beta_{1}$ PreVaccination $_{i, t}+\beta_{2}$ JustVaccinated $_{i, t}+\beta_{3}$ FullyVaccinated $_{i, t}+$ $\beta_{4}$ PostVaccinated $_{i, t}+\alpha_{t}$ Wave $_{t}+\gamma$ Controls $_{i}+\varepsilon_{i, t}$

where JustVaccinate ${ }_{i, t}$ is the treatment dummy for respondent $i$ having just received one or two doses at the time $t$ (i.e., survey-wave $t$ ); FullVaccinated $_{i, t}$ and PostVaccinated $_{i, t}$ are treatment dummies for respondent $i$ having already received a full vaccination course for one wave and two waves, respectively, at wave $t$; and PreVaccination ${ }_{i, t}$ is the dummy for respondent $i$ who is unvaccinated at $t$ but will be "just vaccinated" at $t+1$. The reference group in this event-study are all individuals who are unvaccinated at the corresponding survey wave; for instance, at the wave 2, the groups 1-3 are unvaccinated and serve as the reference for estimating the "just vaccinated" effect for groups 4 and 5, and the "fully vaccinated" effect for group 6. Wave ${ }_{t}$ is the survey wave dummy, equal to 1 when $t=2,3$ (wave-2 and 3), otherwise it is $0\left(\right.$ Wave $\left._{1}=0\right) ; \alpha_{t}$ and $\beta_{k}(t=2,3 ; k=1,2,3,4)$ are parameters to be estimated; other variables are the same as model (1).

In this event-study DiD model, the treatment effects are allowed to vary over the 
event time of vaccination ${ }^{36}$. Modelling dynamic and heterogeneous treatment effects in our data is necessary for understanding the temporal effects of vaccination at different stages of vaccination. By estimating the treatment effects of different stages of vaccination simultaneously across different groups over three waves of survey, this model generates estimates that are comparable across the different stages of vaccination. We also use the pretreatment effect to test for pre-existing differences in trends (i.e., the parallel trends assumption) in this analysis, as per common practice.

We used a fixed-effects regression model to estimate the coefficients of models (1) and (2). Typically, individual-level independent variables which are time-invariant, such as age, gender and other personal characteristics, are differenced out in a fixed-effects model. We employ the Least Squares Dummy Variable (LSDV) approach to estimate our FixedEffects models, which allows us to keep time invariant variables in the models ${ }^{37}$.

Utility model for risk-wellbeing trade-off. We use an experienced utility framework to understand the trade-off people make between well-being and risk in making behavioural decisions during the pandemic:

$E U=f(W, R)$, where $W=f_{w}(X, Y, V, Z)$ and $R=f_{r}(X, Y, V, Z)$

where $W$ is wellbeing, $R$ is perceived risk, $X=\left(x_{1}, x_{1}, \ldots, x_{n}\right)$ is a set of positive behaviours (daily activities, 'normal' by pre-pandemic standards, that generate positive utility), and $Y=$ $\left(y_{1}, y_{1}, \ldots, y_{m}\right)$ is a set of preventative behaviours (protective behaviours that reduce risk at some utility cost), $n$ and $m$ are the number of positive and preventative behaviours considered, respectively, $\mathrm{V}$ is a person's vaccination state dummy ( 1 for vaccinated; 0 otherwise), and $Z$ is a set of control variables for individual characteristics. This experienced utility model considers whether being vaccinated affects people's wellbeing and perceived risk, which may be revealed behaviourally, for example by people engaging in more positive behaviours and forgoing preventative behaviours.

The utility model for wellbeing can be empirically tested using the following model (using two-wave panel data) with happiness as the dependent variable:

$$
\begin{aligned}
& \operatorname{Ln}\left(W_{i, t}\right)=a_{0}+a_{1} \operatorname{Ln}\left(X_{i, t}\right)+a_{2} \operatorname{Ln}\left(X_{i, t}\right) V_{i, t}+a_{3} \operatorname{Ln}\left(X_{i, t}\right) V_{i, t} I_{t}+b_{1} \operatorname{Ln}\left(Y_{i, t}\right)+ \\
& b_{2} \operatorname{Ln}\left(Y_{i, t}\right) V_{i, t}+b_{3} \operatorname{Ln}\left(Y_{i, t}\right) V_{i, t} I_{t}+c_{1} I_{t}+c_{2} V_{i, t}+c_{3} V_{i, t} I_{t}+d Z_{i}+\varepsilon_{i t}
\end{aligned}
$$

where $X_{i, t}$ and $Y_{i, t}$ are vectors of positive and preventative behaviours, respectively, for individual $i$ at time $t, I_{t}$ is a dummy variable, equal to 1 after an individual gets vaccinated $(t$ $=2$ ), otherwise $0(t=1)$, which gives this model a DiD logic (testing the impact of partial or 
full vaccination, and its interaction with behaviour, on wellbeing); $a_{j}, b_{j}, c_{j}$, and $d$ are parameters to be estimated, and $\varepsilon_{i t}$ is an error term. Additional behavioural variables may be added as needed.

The above empirical model can be expressed in the following multiplicative form ${ }^{26}$ :

$W_{i, t}=k * X_{i, t}^{\alpha} * Y_{i, t}^{\beta} * e^{g\left(V_{i, t}\right)} * e^{d Z_{i}+\varepsilon_{i, t}}$

The weighting parameters $\alpha=a_{1}+a_{2} V_{i, t}+a_{3} V_{i, t} I_{t}$ and $\beta=b_{1}+b_{2} V_{i, t}+b_{3} V_{i, t} I_{t}$ capture the moderating effects of vaccination state on how much positive and preventative behaviours, respectively, contribute to wellbeing; $g\left(V_{i, t}\right)=c_{1} I_{t}+c_{2} V_{i, t}+c_{3} V_{i, t} I_{t}$ models the impact of getting vaccinated. For better interpretability, we estimate the model using the twowave panel data (Table S9).

The utility model for perceived risk can be tested using the same model (4) with a change in the dependent variable ( $R_{i, t}$ operationalized by perceived threat of COVID-19).

Finally, we use an analogous model to test how the perceived risk of COVID-19 is constructed, and test how much probability- versus outcome-risk informs overall risk perception of COVID-19, and how these weights change after vaccination. We may do so by replacing $X_{i, t}$ and $Y_{i, t}$ by the perceived probability and consequence of COVID-19 infection respectively in Model (5) (Table S11). 


\section{REFERENCES}

1. Christakis, N. A. Apollo's Arrow: The Profound and Enduring Impact of Coronavirus on the Way We Live. Little Brown, New York (2020).

2. Peltzman, S. The effects of automobile safety regulation. J. Polit. Econ. 83, 677-725 (1975).

3. Hedlund, J. Risky business: safety regulations, risks compensation, and individual behaviour. Injury Prevention 6, 82-90 (2000).

4. Wilde, G. J. S. The theory of risk homeostasis: implication for safety and health. Risk Anal. 2, 209-25 (1982).

5. Traeger, M. W., Cornelisse, V. J., Asselin, J., Price, B., Roth, N. J., Willcox, J., ... \& PrEPX Study Team. Association of HIV preexposure prophylaxis with incidence of sexually transmitted infections among individuals at high risk of HIV infection. JAMA 321, 1380-1390 (2019).

6. Lakdawalla, D., Sood, N., \& Goldman, D. HIV breakthroughs and risky sexual behaviour. Quart. J. Econ. 121, 1063-1102 (2006).

7. Sugiyama, T., Tsugawa, Y., Tseng, C. H., Kobayashi, Y., \& Shapiro, M. F. Different time trends of caloric and fat intake between statin users and nonusers among US adults: gluttony in the time of statins?. JAMA Intern. Med, 174, 1038-1045 (2014).

8. Slovic, P. Perception of risk. Science 236, 280-285 (1987).

9. Slovic, P., Fischhoff, B.. Targeting risks. Risk Anal. 2, 227-34 (1982).

10. Loewenstein, G. F., Weber, E. U., Hsee, C. K., \& Welch, N. Risk as feelings. Psychol. Bull. 127, 267 (2001).

11. Trogen, B., \& Caplan, A. Risk compensation and COVID-19 vaccines. Ann. Intern. Med. 174, 858-859 (2021).

12. WHO Pandemic fatigue - reinvigorating the public to prevent COVID-19. Policy framework for supporting pandemic prevention and management. Copenhagen: WHO Regional Office for Europe; 2020. Licence: CC BY-NC-SA 3.0 IGO (2020).

13. Petherick, A., Goldszmidt, R., Andrade, E.B. et al. A worldwide assessment of changes in adherence to COVID-19 protective behaviours and hypothesized pandemic fatigue. Nat. Hum. Behav. 5, 1145-1160 (2021).

14. SteelFisher, G. K., Blendon, R. J., Caporello, H.. An uncertain public - encouraging acceptance of Covid-19 vaccines. N. Engl. J. Med. 384. 1483-1487 (2021).

15. Lopez Bernal, J., Andrews, N., Gower, C., et al. Effectiveness of Covid-19 vaccines against the B.1.617.2 (delta) variant. N. Engl. J. Med. 385, 585-594 (2021). 
16. Yang, J., Marziano, V., Deng, X. et al. Despite vaccination, China needs nonpharmaceutical interventions to prevent widespread outbreaks of COVID-19 in 2021. Nat. Hum. Behav. 5, 1009-1020 (2021).

17. Huang, B., Wang, J., Cai, J. et al. Integrated vaccination and physical distancing interventions to prevent future COVID-19 waves in Chinese cities. Nat. Hum. Behav. 5, 695-705 (2021).

18. Holtz, D., Zhao, M., Benzell, S. G., Cao, C. Y., Rahimian, M. A., Yang, J., ... Eckles, D., \& Aral, S. Interdependence and the cost of uncoordinated responses to COVID19. Proc. Natl. Acad. Sci. U.S.A. 117, 19837-19843 (2020).

19. Chen, M. K., Chevalier, J. A., \& Long, E. F. Nursing home staff networks and COVID-19. Proc. Natl. Acad. Sci. U.S.A. 118 (1) e2015455118 (2021).

20. Jia, J. S., Lu, X., Yuan, Y., Xu, G., Jia, J., \& Christakis, N. A. Population flow drives spatio-temporal distribution of COVID-19 in China. Nature 582, 389-394 (2020).

21. Fu, F., Christakis, N. A. \& Fowler, J. H. Dueling Biological and Social Contagions. Sci. Rep. 7, 43634 (2017).

22. Jia, J., \& Dyer, J. S. A standard measure of risk and risk-value models. Manage. Sci. 42, 1691-1705 (1996).

23. Jia, J., Dyer, J. S., \& Butler, J. C. Measures of perceived risk. Manage. Sci. 45, 519532 (1999).

24. Weber, E. U., \& Milliman, R. A. Perceived risk attitudes: Relating risk perception to risky choice. Manage. Sci. 43, 123-144 (1997).

25. Benzell, S. G., Collis, A., \& Nicolaides, C. Rationing social contact during the COVID-19 pandemic: Transmission risk and social benefits of US locations. Proc. Natl. Acad. Sci. U.S.A. 117, 14642-14644 (2020).

26. Angrist, J.D., \& Pischke, J.S. Mostly Harmless Econometrics: An Empiricist's Companion, Princeton UP (2009).

27. Lazer, D., Hargittai, E., Freelon, D. et al. Meaningful measures of human society in the twenty-first century. Nature 595,189-196 (2021).

28. Jia, J. S., Khan, U., \& Litt, A. The Effect of Self-Control on the Construction of Risk Perceptions. Manage. Sci., 61, 2259-2280 (2015).

29. Higgins, E. T. Promotion and prevention: Regulatory focus as a motivational principle. In Advances in experimental social psychology, 30, 1-46. Academic Press (1998). 
30. Hikosaka, O. The habenula: From stress evasion to value-based decision-making. Nat. Rev. Neurosci. 11, 503-513 (2010).

31. Fang, H., Wang, L., \& Yang, Y. Human mobility restrictions and the spread of the novel coronavirus (2019-ncov) in China. J. Public Econ. 191, 104272 (2020).

32. Iacus, S. M., King, G., \& Porro, G. Causal inference without balance checking: Coarsened exact matching. Polit. Anal. 20, 1-24 (2012).

33. Hamel, L., et al. KFF COVID-19 Vaccine Monitor. Kaiser Family Foundation (2022). https://www.kff.org/coronavirus-covid-19/dashboard/kff-covid-19-vaccine-monitordashboard/.

34. Jacobson, L.S., LaLonde, R.J., \& Sullivan, D.G. Earnings Losses of Displaced Workers. Am. Econ. Rev., 83, 685-709 (1993).

35. Bailey, M.J., \& Goodman-Bacon, A. The War on Poverty's Experiment in Public Medicine: Community Health Centers and the Mortality of Older Americans. Am. Econ. Rev 105, 1067-1104 (2015).

36. Sun, L., \& Abraham, S. Estimating dynamic treatment effects in event studies with heterogeneous treatment effects. J. Econom. 225, 175-199 (2021).

37. Cameron, A. C., \& Trivedi, P. K. Microeconometrics: methods and applications. Cambridge university press (2005).

Acknowledgements: This research was supported by the Research Grants Council of Hong Kong SAR, China grants C7105-20G, 14505217 (J.S.J.) and National Natural Science Foundation of China grants 72042009, 72074072 (J.J.).

Author contributions: J.J. and Y.Y. contributed equally to this paper. All authors performed research, contributed to study design, and commented on the manuscript. J.S.J. designed the survey and obtained data. Y.Y., J.J., and J.S.J. analysed data; J.S.J., N.A.C., and J.J. wrote the manuscript and directed the research.

Correspondence. Correspondence regarding this article should be addressed to Jayson S. Jia (jiia@,hku.hk)

Data and materials availability: Survey data and code files to replicate the results of the paper will be uploaded online. 
Data Protection and Human Subjects Approval. Ethical approval to use anonymized individual-level survey data for research purposes was granted by the Human Research Ethics Committee of The University of Hong Kong (EA210030). All respondents provided informed consent. We had no access to personal identifying information; the survey company matched data across the panels and provided anonymized identification hashes.

Competing interests: Authors declare no competing interests.

Supplementary Information: Attached. 
Table 1: Group classification based on vaccination states in three waves

\begin{tabular}{|c|c|c|l|}
\hline Group & $\begin{array}{c}\text { Vaccination } \\
\text { state in three } \\
\text { waves }\end{array}$ & $\begin{array}{c}\text { Number of } \\
\text { respondents }\end{array}$ & \multicolumn{1}{|c|}{ Description } \\
\hline Group 1 & $(0,0,0)$ & 427 & Not vaccinated in wave 1, wave 2, and wave 3 \\
\hline Group 2 & $(0,0,1)$ & 58 & $\begin{array}{l}\text { Not vaccinated in wave 1 and wave 2, but } \\
\text { received one dose in wave 3 }\end{array}$ \\
\hline Group 3 & $(0,0,2)$ & 97 & $\begin{array}{l}\text { Not vaccinated in wave 1 and wave 2, but } \\
\text { received two doses in wave 3 }\end{array}$ \\
\hline Group 4 & $(0,1,2)$ & 240 & $\begin{array}{l}\text { Not vaccinated in wave 1, but received one } \\
\text { dose in wave 2 and second dose in wave 3 }\end{array}$ \\
\hline Group 5 & $(0,2,2)$ & 162 & $\begin{array}{l}\text { Not vaccinated in wave 1, but received two } \\
\text { doses in wave 2 }\end{array}$ \\
\hline Group 6 & $(1,2,2)$ & 405 & $\begin{array}{l}\text { Received one dose in wave 1 and second dose } \\
\text { in wave 2 }\end{array}$ \\
\hline Group 7 & $(2,2,2)$ & 778 & Received two doses in wave 1 \\
\hline
\end{tabular}




\section{Figure Legends}

Figure 1. Relationship between survey data and objective data. (a) Number of vaccinated survey respondents and (b) vaccinated population size in the US (CDC data). (c) Average relative level of socioeconomic activity from survey data. and (d) Google mobility index in the US, which measures change in location category visits relative to pandemic baseline. (e) Average relative level of protective behaviour from survey data and (f) daily new COVID-19 cases in the US, which rebounded after the third wave of our survey (indicated by dotted line).

Figure 2. Short-term impact of full vaccination. Here, group 3 (completed full vaccination course by wave 3 , received no doses in waves 1 and 2) is the treatment group, and group 1 (no vaccination over all 3 waves) is the reference group in DiD model (1). The left-hand figure (a) provides a parallel trend test for each scale item, and shows that all behavioural and perceptual measures satisfy the parallel trend assumption except for handwashing. The righthand figure (b) shows the magnitude change in behaviour or perception after group 3 completed a full vaccine course (relative to the unvaccinated group). After full vaccination, respondents' overall recent and planned socioeconomic activiy increased significantly; however, risk-probability perception and overall protective behaviours declined significantly. Each bar and corresponding error line indicate the magnitude of estimated parameter value and $95 \%$ confidence interval based on a DiD model (1) using the coarsened exact matching (CEM) approach.

Figure 3. The dynamics effect of vaccination status on behaviours and perceptions. Estimated coefficients of the event-study DiD model (2). (a) The estimated coefficients for the pre-treatment stage are small and not statistically significantly different from zero for all the measures, implying that the parallel trend assumption is satisfied. Panels b-d show estimated changes in behaviours and perceptions (relative to unvaccinated individuals at the corresponding time) during three observable stages of vaccination: (b) Just vaccinated (with either one or two doses), (c) fully vaccinated (one wave after completing vaccine course), and (d) post-vaccinated (two waves after vaccination completion). At more advanced stages of vaccination, the post-treatment increases in normal socioeconomic behaviours and decline in protective behaviours become progressively greater and more statistically significant. Each bar and corresponding error line indicate the magnitude of estimated parameter value and 95\% confidence interval. All estimated coefficients are represented as percent changes (on the left margin) relative to unvaccinated individuals at the corresponding wave of survey.

Figure 4. Risk-wellbeing trade-off for preventative behaviours and daily activities. We use mediation analysis to test the degree to which different behaviours are motivated by perceived threat (red and green dots), and related to happiness (red dots). The orange coloured shading represents the $95 \%$ CI centred on the regression line. Of preventative behaviours, only staying at home reduces happiness, while others are not significantly related to happiness. Only a subset of daily activities (including social activities, shopping, dining, and travel) contribute positively to happiness. 
Figure 1. Relationship between survey data and objective data.

a Vaccinated Subjects - Survey

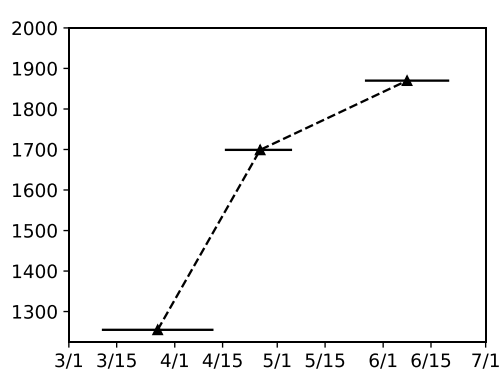

c Daily Activities - Survey

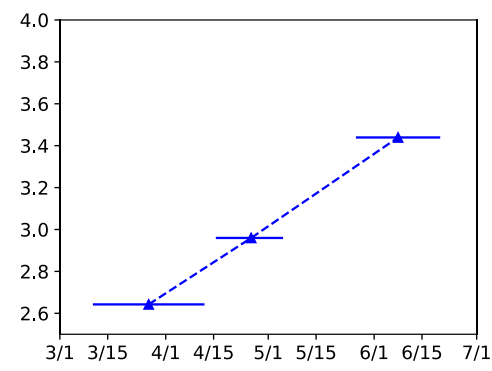

e Protective Behaviors - Survey b Vaccinated Population - CDC

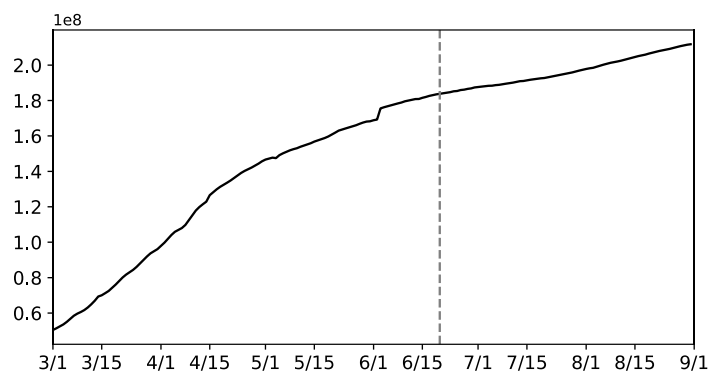

d Google Mobility Index

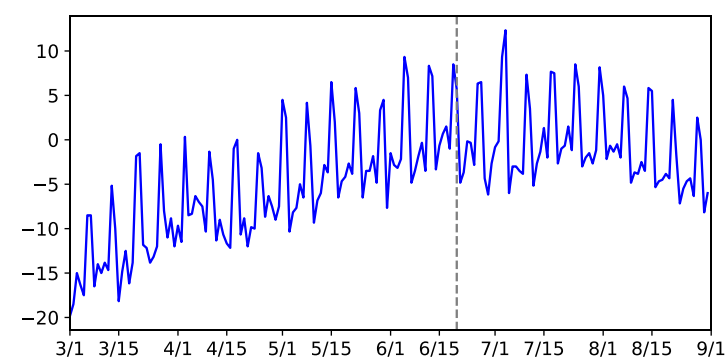

f Daily New Covid-19 Cases

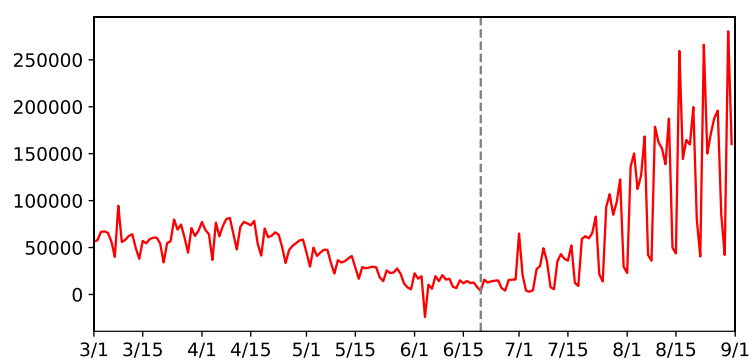


Figure 2. Short-term impact of full vaccination.

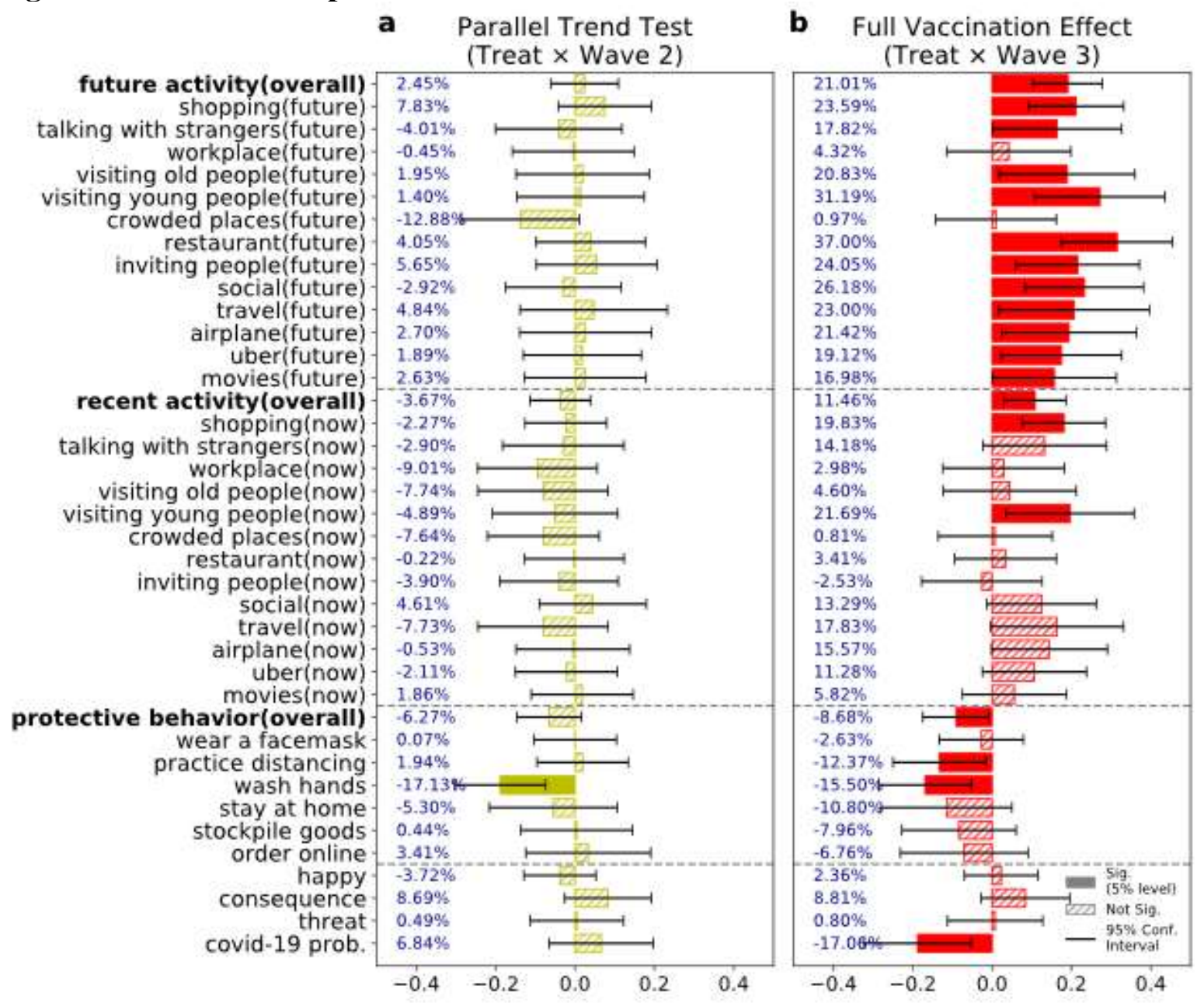


Figure 3. The dynamics effect of vaccination status on behaviours and perceptions.

$$
\text { a Pre-Vaccination }
$$

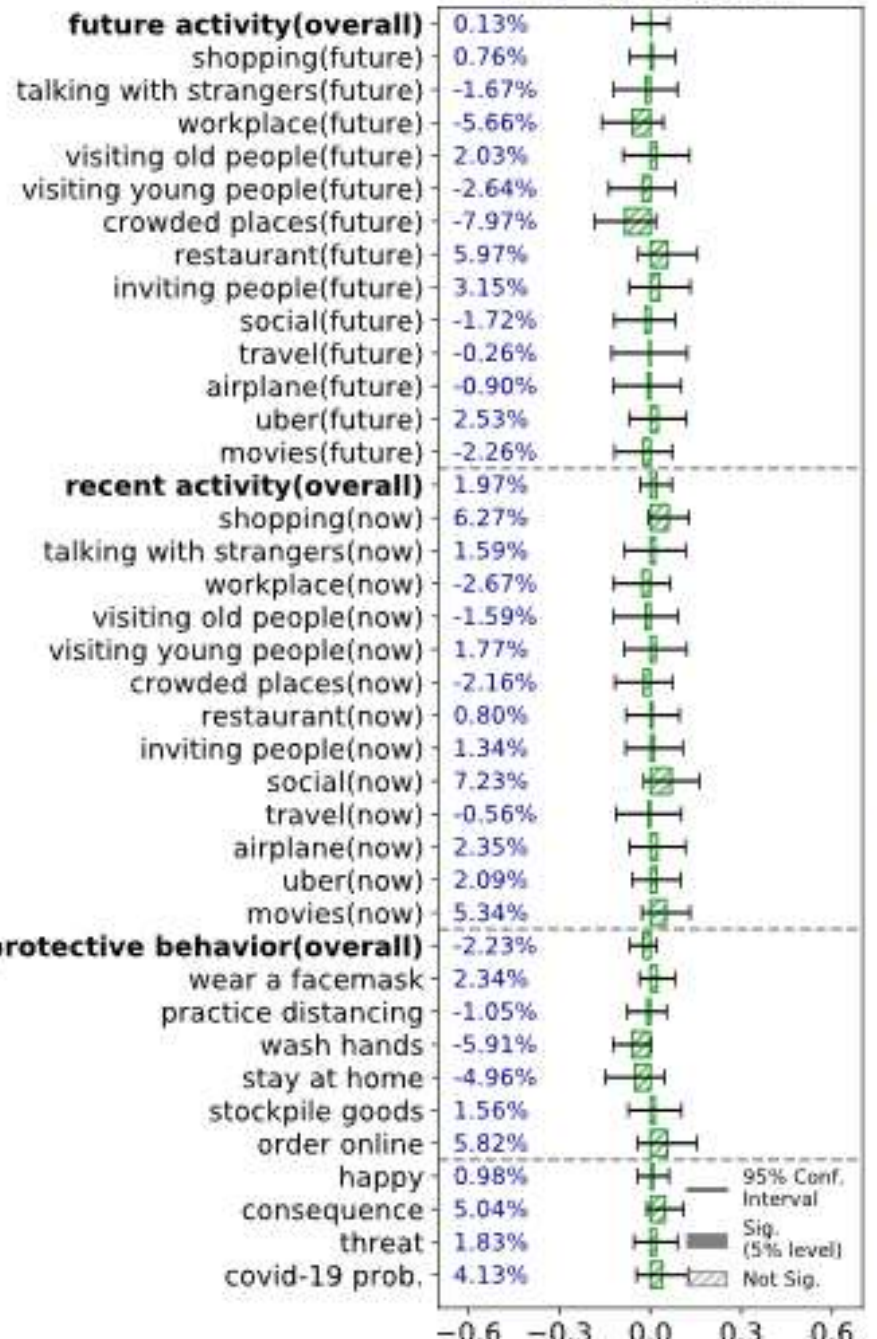

b Just Vaccinated

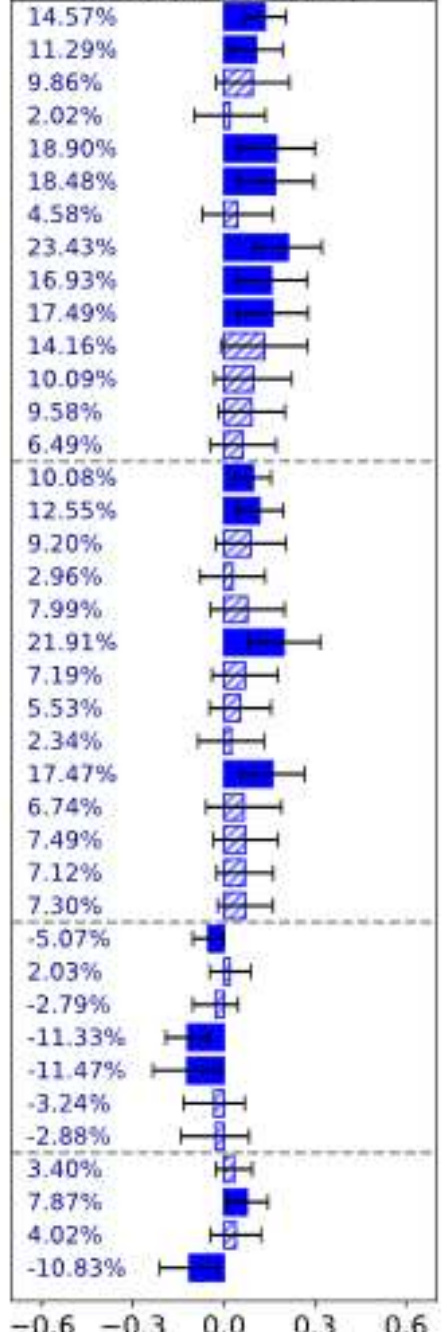

c Fully Vaccinated

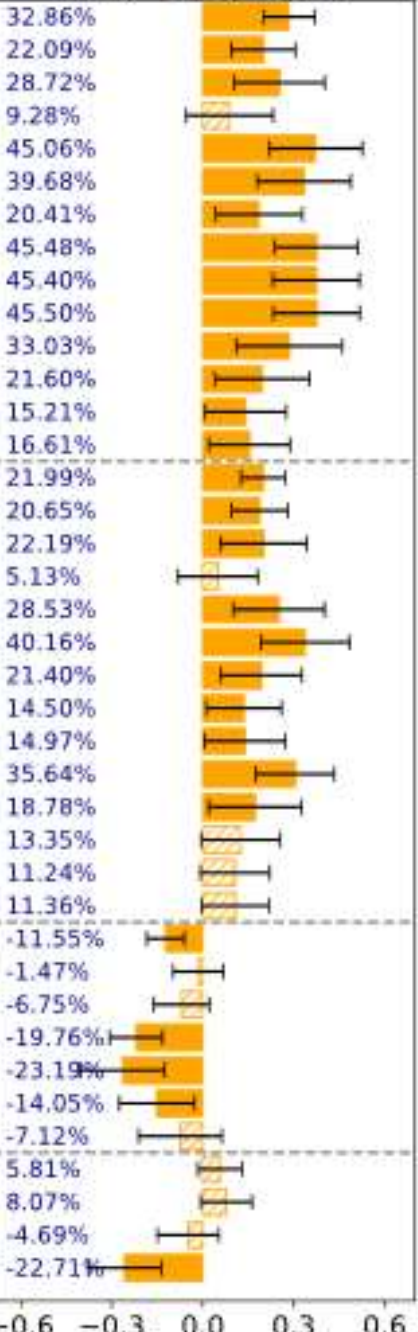

d Post Vaccinated

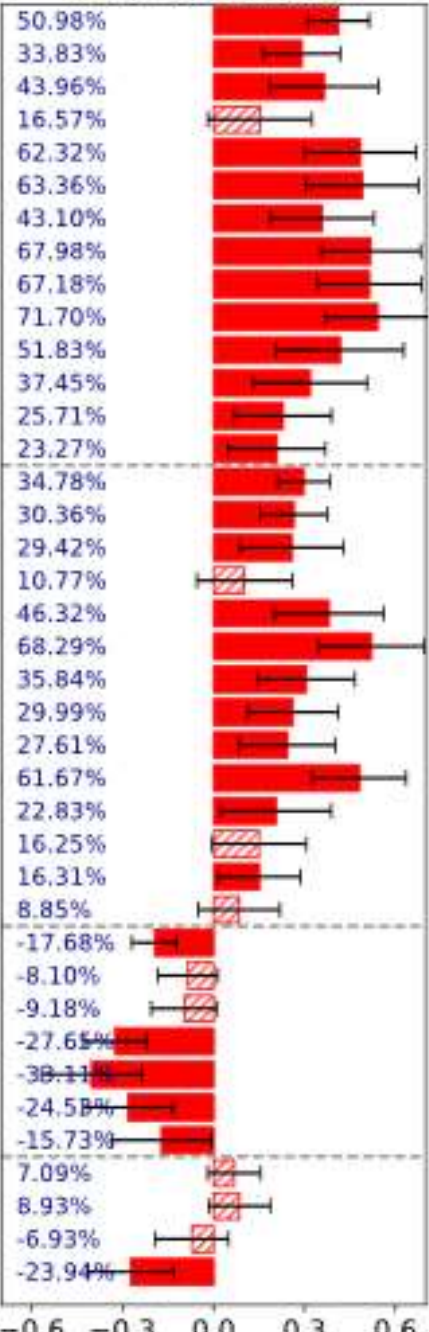


Figure 4. Risk-wellbeing trade-off for preventative behaviours and daily activities.

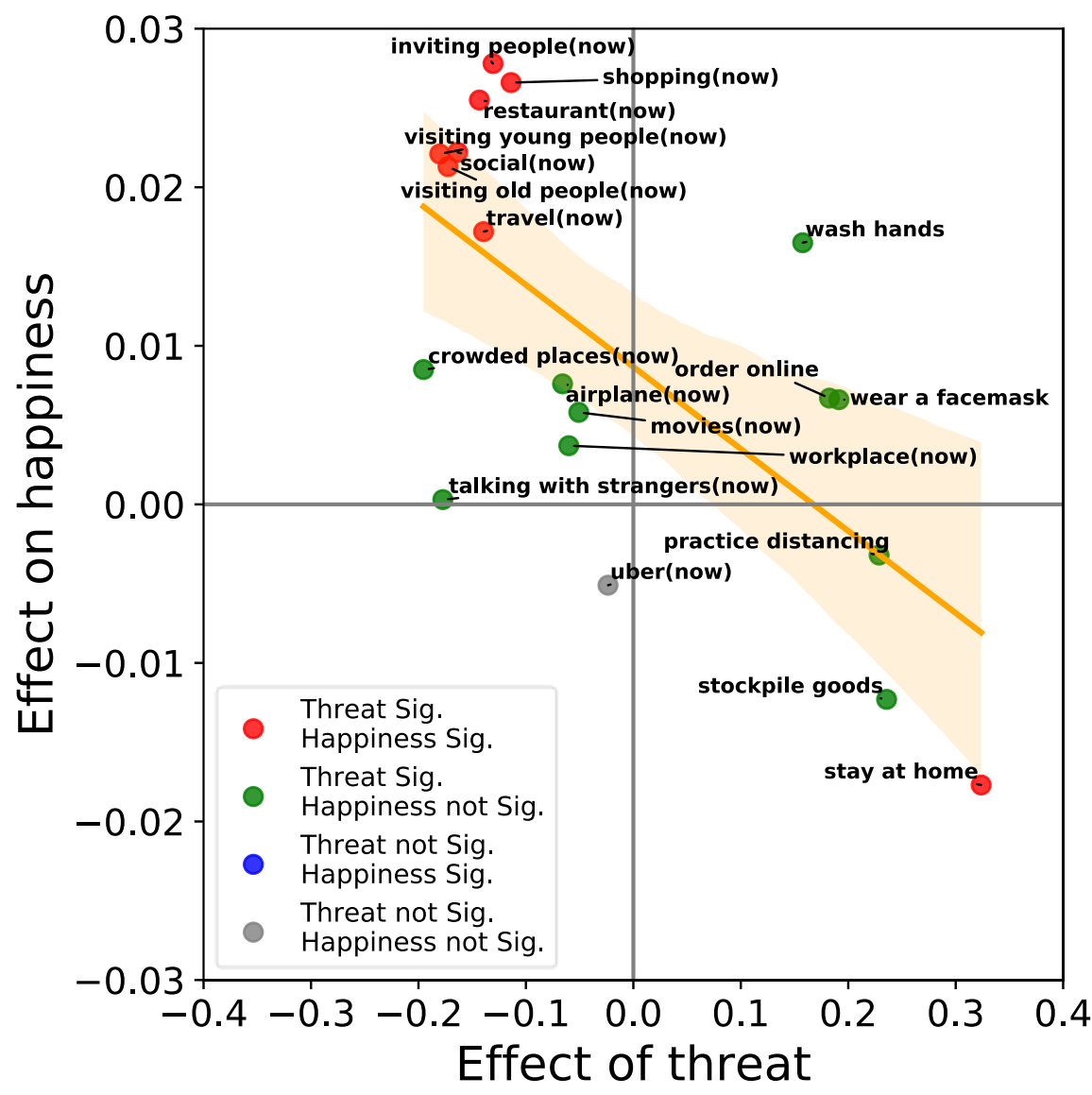

